\title{
Factors affecting urine EIA sensitivity in the detection of Chlamydia trachomatis in men
}

\author{
H Talbot, B Romanowski
}

\begin{abstract}
Objective-This study examined the effects of four variables on the detection of Chlamydia trachomatis in urine from men by enzyme immunoassay (EIA). These variables were: symptoms and signs of urethritis, urine polymorphonuclear leucocytes (PMN), inclusion counts from urethral chlamydia cell cultures and the time between testing and last voiding.

Methods-Included were patients with and without symptoms and/or signs of urethritis attending the Edmonton Sexually Transmitted Disease Clinic. Men were asked to submit a $20 \mathrm{ml}$ volume urine sample. Urethral swabs were collected for gram stain, chlamydia and gonorrhea culture.

Results-A total of 318 men were evaluated of whom 47 had chlamydia. Excluding six men who were coinfected with gonorrhoea, sensitivities and specificities of the Microtrak, Chlamydiazyme and IDEIA systems were $78.1 \%$ and $99.6 \%, 75.6 \%$ and $100 \%$, and $80.5 \%$ and $\mathbf{9 7} \cdot \mathbf{8 \%}$ respectively. Last void time did not affect the sensitivity. However, sensitivity was best when applied to men with severe evidence of urethritis.

Conclusion-There is evidence that urine EIA could be used to detect chlamydia in men with acute urethritis but not in those without signs of urethritis.
\end{abstract}

(Genitourin Med 1994;70:101-104)

\section{Introduction}

Recently published studies evaluating urine as a noninvasive test sample for enzyme immunoassay (EIA) for $C$ trachomatis in males indicate that it may not be suitable in all situations. ${ }^{1-10}$ Jawad et al reported decreased sensitivity of urine EIA in asymptomatic men using the IDEIA method. ${ }^{1}$ Similarly, Hay et al found that IDEIA did not detect organisms when elementary body (EB) counts were low in either the urine or urethra. ${ }^{2}$ Schwebke et al found low inclusion counts in cell culture was a strong predictor of false negative EIA results. ${ }^{3}$ There also may be more chlamydia in an early morning sample than at any other time of day. ${ }^{4}$ Therefore, we evaluated urine EIA sensitivity by four specific variables to explore these points: symptoms and signs of urethritis, numbers of polymorphonuclear leucocytes (PMN) in the urine, inclusion counts from urethral cell cultures, and time between testing and last urination (last void time). Three EIA systems were evaluatedMicrotrak $^{\circledR}$ ) (Syva Company, Palo Alto, CA), Chlamydiazyme ${ }^{\circledR}$ (Abbott Laboratories, North Chicago, Ill) and IDEIA ${ }^{\otimes}$ (bioMerieux API Laboratory Products Limited, Plainview, NY). Chlamydiazyme Blocking Reagent (Abbott) was evaluated as a confirmatory test. The direct immunofluorescence assay (DFA) (Syva) was used in cases of discrepant results between cell culture and urine EIA.

\section{Methods \\ Clinical methods}

The study population included 318 men visiting the Sexually Transmitted Disease (STD) Clinic in Edmonton in 1991.

Included were patients with and without symptoms and/or signs of urethritis. Men submitted a first voided $20 \mathrm{ml}$ urine sample. Three urethral swabs were collected in the following order: smear for Gram stain, swab for chlamydia culture and swab for gonorrhoea culture. The order of urine and swab collection was randomised. Data on symptoms as well as signs of urethral discharge (either spontaneous or easily milked) were recorded. The last void time for each patient was recorded as less than one hour, between one and two hours, between two and three hours or greater than three hours. Exclusion criteria were antibiotic use in the previous four weeks and treatment for non-gonococcal urethritis or gonorrhea in the past three months.

\section{Laboratory methods}

$C$ trachomatis was cultured in cycloheximide treated McCoy cells grown on $12 \mathrm{~mm}$ circular coverslips in one dram shell vials. Fluorescein conjugated monoclonal antibody stain (Syva) was used to identify chlamydial inclusions.

Urines were refrigerated upon collection and processed the same day in the following manner. The urine was vortexed for five seconds and divided into five portions. Three portions were used to perform EIA by the Microtrak, Chlamydiazyme and IDEIA methods. The fourth portion was cultured for chlamdia and the fifth used for a PMN count. The four portions for EIA and chlamydia culture were centrifuged at $2000 \mathrm{~g} \times 25$ minutes in conical polysterene tubes. The supernatant was immediately decanted. Any urine remaining was removed using a pasteur pipette. Pellets for all EIA were then frozen at $-20^{\circ} \mathrm{C}$ and tested within four days. The pellet for
Clinical Investigation Alberta Health, Edmont 
Table 1 Comparison of EIAs for detection of chlamydia in urine specimens

\begin{tabular}{|c|c|c|c|c|c|c|c|c|}
\hline & \multicolumn{4}{|c|}{ No of samples* } & \multicolumn{4}{|l|}{ Per cent $\dagger$} \\
\hline & $T P$ & $T N$ & $F P$ & $F N$ & Sensitivity & Specificity & $P P V$ & $N P V$ \\
\hline $\begin{array}{l}\text { Microtrak EIA } \\
\text { Chlamydiazyme } \\
\text { IDEIA }\end{array}$ & $\begin{array}{l}32 \\
31 \\
33\end{array}$ & $\begin{array}{l}270 \\
271 \\
265\end{array}$ & $\begin{array}{l}1 \\
0 \\
6\end{array}$ & $\begin{array}{l}9 \\
10 \\
8\end{array}$ & $\begin{array}{l}78 \cdot 1 \\
75 \cdot 6 \\
80 \cdot 5\end{array}$ & $\begin{array}{r}99 \cdot 6 \\
100 \cdot 0 \\
97 \cdot 8\end{array}$ & $\begin{array}{r}97 \cdot 0 \\
100 \cdot 0 \\
84 \cdot 9\end{array}$ & $\begin{array}{l}96 \cdot 8 \\
96 \cdot 4 \\
97 \cdot 1\end{array}$ \\
\hline
\end{tabular}

TP, true positive; TN, true negative; FP, false positive; FN, false negative

tPPV, positive predictive value; NPV, negative predictive value.

chlamydia culture was immediately resuspended in $0.5 \mathrm{ml}$ chlamydia 2SP transport media. Urine and urethral swabs were inoculated on the same day of collection to McCoy cells.

To prepare the urine for a PMN count, one-fifth of the original sample was centrifuged at $500 \mathrm{~g} \times 15 \mathrm{~min}$. The supernatant was then decanted except for approximately 1 $\mathrm{ml}$ of urine which was used to resuspend the sediment. A wet mount was prepared with one drop of urine from a pasteur pipette and examined at $400 \times$ magnification. The slide was scanned and five fields, that appeared to have maximum PMN, were selected. Since fields with maximum PMN were always selected this provided a consistent measure of $P M N$ for all patients. The number of PMN in each field was counted to a maximum of 50 . The average number of PMN in the five fields was recorded. This provided an approximate quantization of $P M N$ in the urine.

The usefulness of Chlamydiazyme Blocking Reagent as a confirmatory test was also assessed. For the blocking test, the same urine pellet that had been suspended in Chlamydiazyme EIA specimen buffer and tested was refrozen at $-70^{\circ} \mathrm{C}$ until it was blocked and retested. According to manufacturer's instructions, a specimen was confirmed positive if absorbance fell at least $50 \%$ after addition of blocking reagent. However, if the net absorbance of a previously positive sample was less than $0 \cdot 100$ in the Chlamydiazyme repeat, the result was inconclusive.

In assessing EIA sensitivities, the "gold standard" was a positive cell culture or, in cases of discrepancies, urine DFA positive. For DFA the EIA specimen remnant was spun at $2000 \mathrm{~g} \times 15$ minutes, the supernatant decanted and the pellet resuspended in the liquid remaining. Ten to $20 \mu \mathrm{l}$ of the resuspended pellet was dried on a slide, fixed in methanol and stained. The positive DFA cutoff was one EB. A Chlamydiazyme sample that had an inconclusive blocking result was still considered Chlamydiazyme positive if the patient had a positive cell culture result or had EBs in the urine.

Values of $P$ were calculated by Fisher's exact test ( 2 tail) and by logistic regression.

\section{Results}

Overall the prevalence of $C$ trachomatis in males was $14 \cdot 8 \%(47 / 318)$. Fourteen men had gonorrhoea of whom six were coinfected with chlamydia. Excluding the men with gon- orrhoea, the prevalence of $C$ trachomatis in those with signs of urethral discharge (either a spontaneous or easily milked discharge), was $26 \cdot 1 \%(29 / 111)$. In men with symptoms (of discharge and/or dysuria) but no signs of urethral discharge the prevalence was $9.4 \%$ $(7 \cdot 74)$. In men with no symptoms or signs the prevalence was $4 \cdot 2 \%(5 / 119)$. Since the order of swab and urine collection was randomised, we were unable to define nongonococcal urethritis by PMN count on urethral smear because PMN was washed out of the urethra when urine was collected before swabs.

\section{Laboratory results}

Except where indicated, the 14 men with gonorrhoea are excluded in the results. The average volume of urine collected for chlamydia positive samples was $24 \mathrm{ml}$ urine (range $15-47 \mathrm{ml}$ ). The number of inclusions did not correlate with volume of urine submitted.

The blocking test was carried out on 31 true positive samples. Twenty-seven blocked satisfactorily and four had inconclusive results, that is, had absorbances of less than 0.100 in a previously positive sample.

\section{Test sensitivities}

Table 1 shows a comparison of the three EIA systems. The three tests were not significantly different. Urine cell culture had a sensitivity of $53.6 \%(22 / 41)$ and urethral cell culture had a sensitivity of $95 \cdot 1 \%(39 / 41)$. The urine

Table 2 Predictors of Chlamydia urine EIA sensitivity in males

\begin{tabular}{|c|c|c|}
\hline & & $\begin{array}{l}\text { ELA } \\
\text { Sensitivity } P^{*} \\
(n / N)\end{array}$ \\
\hline \multirow[t]{3}{*}{ Signst } & & \\
\hline & Present & $\begin{array}{l}93.1 \% 0.02 \\
(27 / 29)\end{array}$ \\
\hline & Absent & $\begin{array}{l}58 \cdot 3 \% \\
(7 / 12)\end{array}$ \\
\hline \multirow[t]{3}{*}{ Polymorphonuclear Leucocytes $\ddagger$} & $>4$ & \\
\hline & $=7$ & $\begin{array}{l}90.0 \% 0.000 \\
(25 / 26)\end{array}$ \\
\hline & $<4$ & $\begin{array}{l}60 \cdot 0 \% \\
(9 / 15)\end{array}$ \\
\hline \multirow{2}{*}{ Inclusions§ } & $\geqslant 48$ & $96.2 \% 0.006$ \\
\hline & $<48$ & $\begin{array}{l}60 \cdot 0 \% \\
(9 / 15)\end{array}$ \\
\hline \multirow{3}{*}{ Symptoms $\|$} & Present & $87 \cdot 5 \% 0 \cdot 165$ \\
\hline & & $(28 / 32)$ \\
\hline & Absent & $\begin{array}{l}66 \cdot 7 \% \\
(6 / 9)\end{array}$ \\
\hline
\end{tabular}

* Fisher's exact test ( 2 tail)

† Signs of urethral discharge, spontaneous or easily milked $\neq$ Average number of urine PMN in five fields in one-fifth of the sample

the sample

§ Inclusions in urethral chlamydia cell culture
|| Symptoms of urethral discharge and/or dysuria 
Table 3 Comparison of three ELAs for chlamydia detection in urine by void time and inclusions

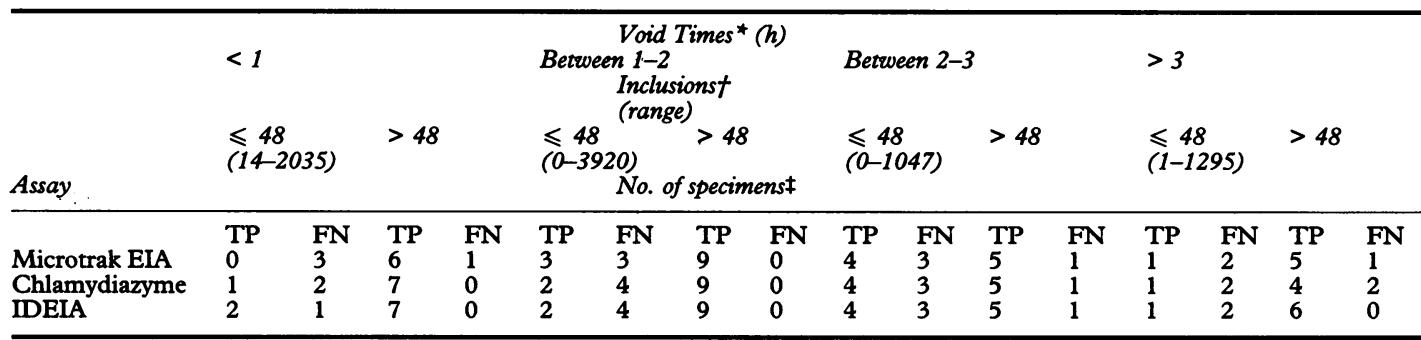

* Number of hours since last voiding

† Number of inclusions in urethral cell culture

$\ddagger$ TP, true positive; FN, false negative.

DFA confirmed two chlamydia urine EIA positives that were not positive by urethral cell culture.

For the six men with chlamydia and gonorrhoea co-infections, the Microtrak, Chlamydiazyme and IDEIA systems detected chlamydia in one, two and three cases respectively. Urethral cell culture detected chlamydia in all six men.

\section{Variables and sensitivity}

To assess EIA sensitivity by symptoms and signs, $P M N$, inclusions, and last void time the results of the three EIA systems were combined. A true positive by any of the three methods was recorded as EIA positive for that sample. Each variable was analysed independently (table 2). Signs of urethral discharge related to detection of chlamydia by both urethral cell culture and urine EIA while absence of signs related to detection of chlamydia by cell culture only $(p=0.02)$. Urine PMN counts of $\geqslant 4$ related to detection of chlamydia by both methods while $<4$ related to

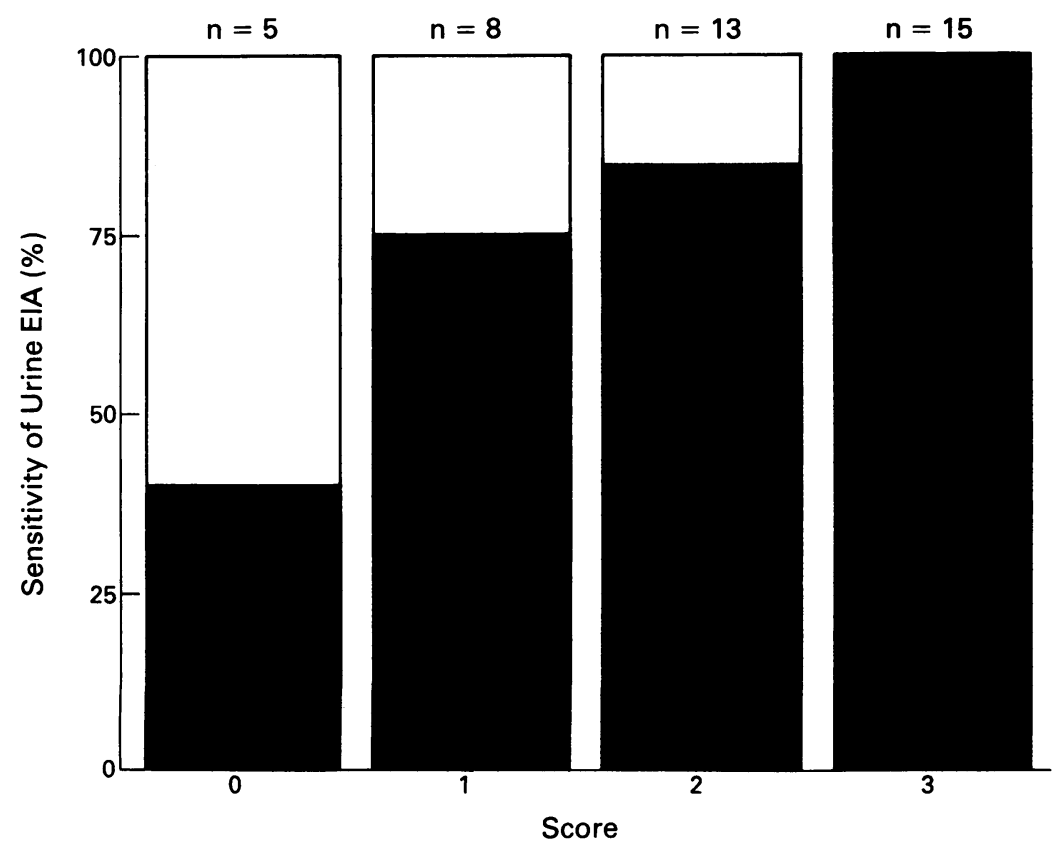

Figure Relationship between symptoms and signs and urine Chlamydia ELA sensitivity in males $\left({ }^{*} p=0.009\right.$ calculated by logistic regression score calculated by allotting one point for: symptoms of urethral discharge, symptoms of dysuria, signs of urethral point for: sym
discharge). detection of chlamydia by cell culture only $(p=$ $0.006)$. Inclusion counts in urethral cell culture of $\geqslant 48$ related to detection of chlamydia by both methods while $<48$ related to detection of chlamydia by cell culture only $(p=$ $0.004)$. The numbers of urethral inclusions in cell culture were low in men who had coinfections with gonorrhea with four of the six having $\leqslant 48$ inclusions. No distinction was observed between men with and without symptoms (note that symptoms and signs were separated in this study) of urethral discharge or dysuria as far as a relationship with EIA sensitivity.

Further analysis was carried out by allotting one point for each of the following: symptoms of urethral discharge, symptoms of dysuria and signs of urethral discharge. Each patient's score was totalled. The figure shows the per cent of positives that EIA detected for scores of $0,1,2$ and 3. Detection for each score was $40.0 \%, 75 \cdot 0 \%, 84.6 \%$, and $100.0 \%$ respectively $(\mathrm{p}=0.009)$.

Inclusion counts in urethral cell culture also related positively with PMN counts in the urine. For inclusion counts $\leqslant 42(n=15)$, the average PMN count was 2.67 in comparison to inclusion counts $>42(n=26)$ where the average PMN count was $15 \cdot 66$.

Table 3 shows that each void time category had a wide range of inclusions and comparable numbers of undetected chlamydia were found in all void time categories for men with $\leqslant 48$ inclusions.

\section{Discussion}

Several studies have been done to evaluate urine EIA to test for chlamydia in males. In high prevalence populations published sensitivities for Chlamydiazyme are $86 \cdot 8 \%, 84 \cdot 2 \%$, $77 \cdot 8 \%$ and $77 \cdot 0 \%$ and sensitivities for IDEIA are $81 \cdot 6 \%$ and $100 \cdot 0 \% .^{5-6}$. Except for the last mentioned study, we consider the sensitivities to be unsatisfactory.

It was not specified in the above studies if the patients with false negative urine EIA had urethritis. Other studies have provided further information in this regard. Hay et al, tested only men with acute urethritis and found IDEIA had a sensitivity of $85 \% .^{2}$ When IDEIA did not detect chlamydia it was in samples with less than 10 elementary bodies in the urine or urethra. Jawad et al found the 
sensitivity of IDEIA was $79 \cdot 7 \%$ in men with urethritis. The sensitivity dropped to $59 \%$ in those without clinical urethritis. ${ }^{1}$ Jensen compared urine with urethral swabs in a largely asymptomatic population and found sensitivities of $82 \cdot 1 \%$ and $62 \cdot 1 \%$ for Microtrak EIA and Chlamydiazyme respectively..$^{10}$ Schwebke et al had a study population in which $54 \%$ had urethritis and found a sensitivity for Chlamydiazyme of only $42 \%$ compared with urethral cell culture. ${ }^{3}$ In contrast, Leonardi et al found sensitivities of $79.1 \%, 91.7 \%$ and $\mathbf{9 5 . 8 \%}$ for Chlamydiazyme, IDEIA and Microtrak EIA in an asymptomatic population. ${ }^{11}$

We analysed our data further by correlating EIA results with symptoms and signs of urethral discharge. There was a stepwise increase in sensitivity as the patient had increasingly more evidence of urethritis. Further analysis demonstrated that if the patient had signs of urethral discharge or $\geqslant 4 \mathrm{PMN}$ in the urine sample, the urine EIA was a reliable alternative to culture of urethral swabs.

Inclusion counts from cell culture related positively with EIA sensitivity and with urine PMN. Urine EIA sensitivity was high when the number of inclusions was $\mathbf{4 8}$ or greater in urethral cell culture. As in our study, Schwebke et al suggested a strong link between low inclusion counts and poor urine EIA sensitivity. ${ }^{3}$

Urine EIA had poor performance when used for men who were coinfected with gonorrhoea. These men also had low numbers of inclusions in urethral cell cultures. It is unclear how these factors related.

The sensitivities of the urine EIA tests need to be improved in men without acute urethritis. We feel sensitivities should approach $90 \%$ before urine can routinely replace urethral swabs. If equally high sensitivities cannot be reached in asymptomatic populations, there will be false assurance of a chlamydia free population. The presence of $P M N$ in urine was an indicator of sensitivity in this study. The use of PMN in urine as a screening test has been explored by others with variable results. ${ }^{12-15}$ Although our numbers were small, longer void times did not improve sensitivity in cases of low inclusions. A study by Thomas et al also suggested that an early morning urine sample was equally sensitive to a first passed sample at any other time. ${ }^{16}$

In conclusion there is evidence that urine EIA could be used to detect chlamydia in men with acute urethritis but not in those without signs of urethritis.

We thank Syva, Abbott and bioMarioux API for donating reagents for this project. We also thank Michel Joffres for statistical analysis of the data.

1 Jawad AJ, Manuel G, Matthews R, Wise R, Clay J. Evaluation of a genus-specific monoclonal antibody in amplified enzyme-linked immunoassay in the detection amplified enzyme-linked immunoassay in the detection of chlamydia in

2 Hay PE, Thomas BJ, Gilchrist C, Palmer HM, Gilroy CB, Taylor-Robinson $D$. The value of urine samples from men with non-gonococcal urethritis for the detection of Chlamydia trachomatis. Genitourin Med 1991;67:124-8.

3 Schwebke JR, Clark AM, Pettinger MB, Nsubga $P$ Stamm WE. Use of urine ezyme immunoassay as a diag nostic tool for Chlamydia trachomatis urethritis in men. Clin Microbiol 1991;29:2446-9.

4 Matthews RS, Wise R. Non-invasive sampling method for detecting Chlamydia trachomatis. (letter) Lancet 1989, i:96-7.

5 Chernesky M, Castriciano S, Sellors J, et al. Detection of Chlamydia trachomatis antigens in urine as an alternative to swabs and cultures. $¥$ Infect Dis 1990;161:124-6.

6 Paul ID, Caul EO. Evaluation of three Chlamydia tra chomatis immunoassays with an unbiased, noninvasive chomatis immunoassays with an unbiased, non

7 Mumtaz G, Ridgway GL, Clark S, Allason-Jones E. Evaluation of an enzyme immunoassay (Chlamydiazyme) with confirmatory test for the detection of chlamydial antigen in urine from men. Int $\mathcal{F} S T D$ and Aids 1991;2:359-61.

8 Genc M, Stary A, Bergman S, Mardh P. Detection of Chlamydia trachomatis in first-void urine collected from men and women attending a venereal clinic. APMIS 1991;99:455-9.

9 Ferris DG, Martin WH, Mathis DM, Steel JCH, Fisher PM, Styslinger KM. Noninvasive detection of Chlamydia trachomatis urethritis in men by a rapid enzyme immunoassay test. 7 Fam Pract 1991;33:73-8.

10 Jensen IP. A comparison of urine sample to urethral swab for detection of Chlamydia trachomatis in asymptomatic young men using two enzyme immunoassays. Sex Transm Dis 1992;19:165-9.

11 Leonardi GP, Seitz M, Edstrom R, Cruz J, Costello P, Szabo K. Evaluation of three immunoassays for detection of Chlamydia trachomatis in urine specimens from tion of Chlamydia trachomatis in urine specimens from
asymptomatic males. $¥$ Clin Microbiol 1992;30:2793-6.

12 White DJ, Malet RM, Bignell CJ. Non-invasive samplin White DJ, Malet RM, Bignell CJ. Non-invasive sampling
method for detecting Chlamydia trachomatis. (letter) Lancet 1989;i:96-7.

13 Bowie WR. Comparison of gram stain and first-voided urine sediment in the diagnosis of urethritis. Sex Trans Dis 1978;5:39-42.

14 Braverman PK, Biro FM, Brunner RL, Gilchrist MJR Rauh JL. Screening asymptomatic adolescent males for chlamydia. F Adolesc Health Care. 1990;11:141-4.

15 Adger H, Sweet RL, Shafer M, Schachter J. Screening for Chlamydia trachomatis and Neisseria gonorrhoeae in adolescent males; value of first catch urine examination. Lancet 1984;ii:944-5.

16 Thomas BJ, Gilchrist C, Hay PE, Taylor-Robinson D Simplification of procedures used to test urine samples for Chlamydia trachomatis. $¥$ Clin Pathol 1991;44:374-5. 